\title{
Correction to: The Procedureless Elipse Gastric Balloon Program: Multicenter Experience in 1770 Consecutive Patients
}

R. lenca ${ }^{1} \cdot$ Mohammed Al Jarallah $^{2}$ - Adelardo Caballero ${ }^{3} \cdot$ Cristiano Giardiello $^{4} \cdot$ Michele Rosa $^{5} \cdot$ Sébastien Kolmer $^{6}$. Hugues Sebbag ${ }^{7}$. Julie Hansoulle ${ }^{8}$. Giovanni Quartararo ${ }^{9}$. Sophie Al Samman Zouaghi ${ }^{10} \cdot$ Girish Juneja $^{11}$. Sébastien Murcia ${ }^{12} \cdot$ Roman Turro $^{13} \cdot$ Alberto Pagan $^{14} \cdot$ Faruq Badiuddin $^{15} \cdot$ Jérôme Dargent $^{16} \cdot$ Pierre Urbain $^{17}$. Stefan Paveliu ${ }^{18} \cdot$ Rita Schiano di Cola ${ }^{4}$. Corrado Selvaggio ${ }^{5} \cdot$ Mohammed Al Kuwari $^{19}$

Published online: 5 May 2020

(C) Springer Science+Business Media, LLC, part of Springer Nature 2020

\section{Correction to: Obesity Surgery} https://doi.org/10.1007/s11695-020-04539-8

An author name was incorrectly indicated in the Conflict of Interest Statement. The correct statement follows:

\section{Conflict of Interest}

Roberta Ienca and Cristiano Giardiello are consultants for Allurion; F. Badiuddin is an advisor for Allurion. The authors declare that there are no other conflicts of interest for this study.

Publisher's note Springer Nature remains neutral with regard to jurisdictional claims in published maps and institutional affiliations.

The online version of the original article can be found at https://doi.org/ $10.1007 / \mathrm{s} 11695-020-04539-8$

R. Ienca

roberta.ienca@gmail.com

Extended author information available on the last page of the article 


\section{Affiliations}

\section{R. lenca ${ }^{1} \cdot$ Mohammed Al Jarallah $^{2} \cdot$ Adelardo Caballero $^{3} \cdot$ Cristiano Giardiello $^{4} \cdot$ Michele Rosa $^{5} \cdot$ Sébastien Kolmer $^{6}$. Hugues Sebbag ${ }^{7}$. Julie Hansoulle ${ }^{8}$. Giovanni Quartararo ${ }^{9}$. Sophie Al Samman Zouaghi ${ }^{10} \cdot$ Girish Juneja $^{11}$. Sébastien Murcia ${ }^{12} \cdot$ Roman Turro ${ }^{13} \cdot$ Alberto Pagan $^{14} \cdot$ Faruq Badiuddin $^{15}$. Jérôme Dargent ${ }^{16} \cdot$ Pierre Urbain $^{17}$. Stefan Paveliu ${ }^{18} \cdot$ Rita Schiano di Cola ${ }^{4} \cdot$ Corrado Selvaggio $^{5} \cdot$ Mohammed Al Kuwari $^{19}$}

Mohammed Al Jarallah

jarallah55@hotmail.com

\author{
Adelardo Caballero \\ info@institutodeobesidad.com \\ Cristiano Giardiello \\ cristiano.giardiello@pinetagrande.it \\ Michele Rosa \\ michelerosa@tiscali.it \\ Sébastien Kolmer \\ seb.kolmer@yahoo.fr \\ Hugues Sebbag \\ hsebbag.ppr@hotmail.fr \\ Julie Hansoulle \\ j.hansoulle@ clarisclinic.com \\ Giovanni Quartararo \\ giovanni.quartararo@gmail.com \\ Sophie Al Samman Zouaghi \\ dralsammanzouaghi@gmail.com \\ Girish Juneja \\ gijuneja@gmail.com
}

1 Weight Management Center, Nuova Villa Claudia Clinic,

Rome, Italy

2 General Surgery Department, Jarallah German Clinic, Kuwait City, Kuwait

3 Bariatric Center, Instituto De Obesidad, Madrid, Spain

4 Emergency and Metabolic Surgery Department, Pineta Grande Hospital, Caserta, Italy

5 Nutritional Center, Micros Clinic, Modica, Italy

6 Digestive Surgery Department, Le Réseau Pondera, Mulhouse, France

7 Digestive Surgery Department, Polyclinique Du Parc Rambot, Aixen-Provence, France

8 Nutrional Center, Claris Clinic, Brussels, Belgium

9 General and Bariatric Surgery Unit, Villa Donatello, Florence, Italy

10 Digestive and Bariatric Surgery Department, Infirmerie Protestante, Caluire, France

11 Bariatric and Weight Loss Center, Cocoona Center, Dubai, UAE
Sébastien Murcia

sebastienmurcia@wanadoo.fr

Roman Turro

romanturro@gmail.com

Alberto Pagan

albertopagan@me.com

Faruq Badiuddin

faruq1@gmail.com

Jérôme Dargent

jerome.dargent@polyclinique-rillieux.fr

Pierre Urbain

urbain@ chirobes.com

Stefan Paveliu

spaveliu2000@gmail.com

Rita Schiano di Cola

rita_schiano@libero.it

Corrado Selvaggio

selvaggio.corrado@virgilio.it

Mohammed Al Kuwari

drmalkuwari@gmail.com

12 Bariatric Surgery Department, Nouvelle Clinique Bordeaux Tondu, Floirac, France

13 Digestive Endoscopy Department, Centro Medico Teknon, Barcelona, Spain

14 Nutritional Center, Centro Integral Nutricion Baleares-Cinib, Palma de Mallorca, Spain

15 General and Obesity Surgery Department, BR Medical Suites, Dubai, UAE

16 Bariatric Surgery Department, Polyclinique Lyon Nord, Rillieux-laPape, France

17 General Surgery Department, Polyclinique Saint Privat, Boujansur-Libron, France

18 General and Obesity Surgery Department, Centre Medical Matisse, Nice, France

19 Bariatric Surgery Department, The Masters Medical Clinic, Doha, Qatar 